Ney Francisco Pinto Costa ${ }^{1}$

Elisabeth Anhel Ferraz ${ }^{2}$

Cynthia Teixelra de Souza ${ }^{3}$

Cláudio Felipe Ribero da Silva ${ }^{4}$

MÔNICA Gomes de AlmeidA ${ }^{5}$

Artigos originais

Palavras-chave

Anticoncepção/métodos Planejamento familiar Saúde sexual e reprodutiva Acesso à informação

Keywords

Contraception/methods Family planning (Public Health) Sexual and reproductive health Access to information

\section{Acesso à anticoncepção de emergência: velhas barreiras e novas questões}

\author{
Access to emergency contraception: old barriers and new questions
}

\section{Resumo}

OBJETIVO: comparar duas estratégias de acesso à anticoncepção de emergência (AE): informação e informação com entrega antecipada do método, e sua relação com o uso deste método e com o uso regular de anticoncepcionais. MÉTODOS: de agosto de 2004 a janeiro de 2005, foram recrutadas voluntárias de 18 a 49 anos, atendidas em clínicas de saúde reprodutiva em seis cidades brasileiras. Os sujeitos foram distribuídos aleatoriamente no grupo que recebeu informação (Grupo Controle) ou no grupo que recebeu informação e entrega antecipada (Grupo Medicado). Houve visitas de seguimento em quatro e oito meses. Para a análise estatística, foram usados os testes de Person e McNemar. RESULTADOS: dos 823 sujeitos recrutados, 407 completaram os oito meses de observação e constituíram a amostra para análise. A maioria dos sujeitos (61\%) não usou AE. Os sujeitos do Grupo Medicado usaram mais AE (57\%) do que os do Grupo Controle (18\%), e o fizeram mais precocemente em relação ao tempo desde a relação sexual desprotegida. Houve aumento significativo no uso regular de anticoncepcionais entre os sujeitos que usaram AE do Grupo Medicado (88\% versus 97\%) e uma redução sem significado estatístico no Grupo Controle. CONCLUSÕES: informação e entrega antecipada ampliaram o acesso e uso da AE e não reduziram o uso regular de anticoncepcionais, incluindo preservativos.

\section{Abstract}

PURPOSE: to compare two strategies of access to emergency contraception: only information and information with previous delivery of this contraceptive method, and its relationship with the use of this method and the regular use of contraceptives. METHODS: from August 2004 to January 2005, 18 to 49-year-old volunteers, attended at reproductive health clinics from six Brazilian towns were recruited. The subjects were randomly distributed in a group getting information about emergency contraception (Control Group), or in a group getting information about this method and previous delivery of the contraceptive (Medicated Group). Follow-up visits occurred into four and eight months. Person and McNemar's tests were used for the statistical analysis. RESULTS: from the 823 recruited subjects, 407 completed the 8-month-observation period and were the sample analyzed. Most of the subjects (61\%) did not use the emergency contraceptive. The subjects from the Medicated Group used more emergency contraceptives (57\%) than the ones from the Control Group (18\%), and they did it more precociously, concerning the time since the unprotected sexual intercourse. There was a significant increase of regular use of contraceptives among the subjects who used emergency contraceptives in the Medicated Group (88\% versus $97 \%$ ) and a statistically nonsignificant decrease in the Control Group. CONCLUSIONS: information and previous delivery intensified the access and use of emergency contraceptives, and did not reduce the regular use of contraceptives, including condoms. (EP 20031-170 - Rio de Janeiro/R Fone: (21) 3861-2439/ Fax: (21) 3861-2469 E-mail: monica@bemfam.org.br

Recebido 07/08/2007

Aceito com modificacọ̃es
Trabalho realizado pela Bem Estar Familiar no Brasil - BEMFAM - Rio de Janeiro (RJ), Brasil.

' Secretário Executivo da Bem Estar Familiar no Brasil - BEMFAM - Rio de Janeiro (RJ), Brasil.

${ }^{2}$ Coordenadora do Departamento de Pesquisas Sociais da Bem Estar Familiar no Brasil - BEMFAM - Rio de Janeiro (R)), Brasil.

3 Pesquisadora da Bem Estar Familiar no Brasil - BEMFAM - Rio de Janeiro (RI), Brasil.

${ }^{4}$ Estatístico da Bem Estar Familiar no Brasil - BEMFAM - Rio de Janeiro (RJ), Brasil.

${ }^{5}$ Coordenadora do Departamento Médico da Bem Estar Familiar no Brasil - BEMFAM - Rio de Janeiro (RJ), Brasil.

Apoio financeiro: da Bergstrom Foundation, Califórnia - USA. Apoio Técnico da Federação Internacional de Planejamento Familiar (IPPF), New York - USA 


\section{Introdução}

A anticoncepção de emergência (AE) é um método hormonal indicado para evitar gravidez depois de uma relação sexual desprotegida, quando houver falha no uso de método anticoncepcional ou em casos de violência sexual $^{1}$. A anticoncepção oral de emergência pode atuar por meio da inibição ou retardo da ovulação, alteração do transporte dos espermatozóides e do óvulo, modificação do muco cervical e interferência na capacitação espermática. Sua ação se faz, portanto, antes da fecundação. Caso seja utilizada após a implantação, não interrompe gravidez em curso $^{2}$. Apesar da eficácia da AE ser conhecida há mais de 30 anos, constitui-se numa opção ainda pouco utilizada para redução da gravidez não planejada e da morbimortalidade associadas ao abortamento inseguro, principalmente em países da América Latina ${ }^{3}$.

Com o objetivo de ampliar o acesso e uso deste método, em 1995, sete instituições, entre as quais a Organização Mundial de Saúde (OMS) formaram o Consórcio Internacional de Anticoncepção de Emergência. No mesmo ano, o método passou a integrar a lista de medicamentos essenciais da OMS. No que se refere ao contexto nacional, podem ser destacadas duas datas: 1996, com a inclusão da AE na norma técnica sobre anticoncepção do Ministério da Saúde, e 1999, com a comercialização de um produto específico no mercado brasileiro. Os métodos anticoncepcionais de emergência disponíveis e recomendados pelo Ministério da Saúde são: produto específico (pílulas de levonorgestrel) e método Yuzpe (pílulas combinadas comuns usadas em doses especiais).

A anticoncepção hormonal de emergência é eficaz até cinco dias após a relação sexual desprotegida, sendo tanto mais eficaz quanto mais precocemente for usada. Devido a esta particular característica de uso, é importante que as mulheres tenham condições de acesso rápido ao método, nos casos indicados. $\mathrm{O}$ acesso à $\mathrm{AE}$ pode ser dificultado por vários tipos de barreiras, tais como falta de informação, incluindo a percepção equivocada de que o método poderia ser abortivo, aspectos morais e culturais, preço dos produtos e a exigência de prescrição médica ${ }^{4,5}$. Outra barreira relevante é a preocupação dos profissionais quanto ao potencial negativo que a informação mais ampla sobre o método poderia ter sobre o uso regular de anticoncepcionais, em especial preservativos ${ }^{6-9}$. Apesar desta preocupação não ter sido confirmada por estudos realizados em outros países ${ }^{10-14}$, a falta de estudos nacionais segue sendo uma barreira para que muitos profissionais ampliem a prescrição da AE.

O trabalho aqui exposto é um estudo longitudinal, que tem como objetivo comparar duas estratégias de acesso à $\mathrm{AE}$ no contexto brasileiro (AE) - informação com entrega antecipada do método e somente informação - considerando o padrão de uso da $\mathrm{AE}$ e os efeitos do acesso à $\mathrm{AE}$ sobre o uso regular de anticoncepcionais, incluindo preservativos.

\section{Métodos}

A pesquisa foi realiazada pela BEMFAM nos Estados do Rio de Janeiro, Ceará; Maranhão, Paraíba, Pernambuco e Rio Grande do Norte. Entre agosto de 2004 e janeiro de 2005, foram recrutadas 823 voluntárias em seis clínicas de saúde reprodutiva da organização não governamental Bem Estar Familiar no Brasil (Bemfam), localizadas nas cidades de São Luís, Fortaleza, Natal, João Pessoa, Recife e Rio de Janeiro. Os critérios de inclusão foram: mulheres que buscaram atendimento ginecológico com idade entre 18 e 49 anos; com atividade sexual (que tiveram relações sexuais nas últimas quatro semanas); mulheres que não estavam grávidas nem desejavam engravidar nos próximos seis meses. Foram excluídas do estudo as mulheres submetidas à ligadura de trompas, usuárias de dispositivo intra-uterino (DIU) e aquelas com suspeita de gravidez ou que desejassem engravidar nos próximos seis meses.

A pesquisa e seus instrumentais foram submetidos $\mathrm{e}$ aprovados pelo Comitê de Ética em Pesquisa da Bemfam e as voluntárias, após receberem todas as informações sobre a pesquisa, assinaram um termo de consentimento livre e esclarecido.

Em cada clínica, foram formados aleatoriamente dois grupos: Grupo Medicado (informação e entrega antecipada) e Grupo Controle (somente informação). Todas as mulheres (Grupos Medicado e Controle) receberam informações sobre a AE, uso de preservativos e prevenção de doenças sexualmente transmissíveis (DST), incluindo a infecção pelo vírus da imunodeficiência humana (HIV), e receberam material impresso sobre o método. O período de observação do estudo foi de oito meses, com a realização de três entrevistas: no recrutamento, quatro e oito meses após.

Após a entrevista médica para recrutamento, as mulheres do Grupo Medicado receberam dois kits compostos cada um por duas doses do produto específico (quatro comprimidos de $0,75 \mathrm{mg}$ de levonorgestrel), um preservativo masculino e um folheto sobre a $\mathrm{AE}$. As mulheres do Grupo Controle receberam também, no momento da entrevista, dois kits, cada um contendo um preservativo masculino e um folheto sobre a AE.

É importante salientar que todas as participantes da pesquisa, independentemente se pertencentes ao Grupo Medicado ou ao Grupo Controle, foram informadas que, se porventura houvesse necessidade, poderiam retornar às clínicas da Bemfam para receberem $\mathrm{AE}$ a qualquer momento. Esta demanda foi caracterizada como extra e registrada em instrumental próprio.

Para a coleta das informações nas distintas fases da pesquisa, foram utilizados questionários específicos. Na primeira fase (recrutamento) foi empregado o questionário A1, que 
abarcava dados sociodemográficos, assim como informações sobre reprodução e anticoncepção, permitindo a identificação do perfil das entrevistadas. Nas demais fases - denominadas de primeira visita de seguimento e de segunda visita de seguimento - os questionários S1 e S2 foram usados no regresso dos sujeitos às clínicas, com o objetivo de atualizar o grupo participante, assim como suas informações. Para saber o tipo de instrumental, questionário $\mathrm{S} 1$ ou $\mathrm{S} 2$, a ser respondido durante as visitas de seguimento da pesquisa, perguntava-se à participante se ela fez uso da $\mathrm{AE}$ nos períodos observados (entre o recrutamento e a primeira visita de seguimento e entre a primeira visita de seguimento e a segunda visita). Caso a resposta fosse positiva, a voluntária respondia às perguntas do questionário $\mathrm{S} 1$, elaborado para as mulheres que fizeram uso da AE. Caso a participante não tivesse feito uso, o questionário S2, elaborado para aquelas que não utilizaram o método durante o período em questão, era respondido. Também, durante as visitas de regresso às clínicas, caso houvesse necessidade, insumos e materiais educativos eram disponibilizados para as participantes do estudo.

Para a verificação de diferenças estatisticamente significativas entre variáveis quantitativas, foi utilizado o teste do $\chi^{2}$ de Pearson. Para testar a significância das mudanças ocorridas no uso regular da anticoncepção antes e após o uso da $\mathrm{AE}$, utilizou-se o teste de $\mathrm{McNemar}$, em função da análise de amostras pareadas e em dois momentos de observação ${ }^{15}$.

Não houve incentivos financeiros para participação no estudo. Como uma estratégia para minimizar as perdas, ofereceu-se a cada participante, no retorno da primeira visita de seguimento, uma ajuda de custo de $\mathrm{R} \$ 10,00$ (dez reais) e, para aquelas que retornavam para a segunda visita de seguimento, uma consulta ginecológica e uma citologia cervical sem custos, se necessário.

\section{Resultados}

Nas seis clínicas, foram recrutadas 823 voluntárias, sendo 419 aleatoriamente alocadas no Grupo Medicado e 404 no Grupo Controle. Das 823 voluntárias recrutadas, 407 (49,5\%) completaram oito meses de observação, sendo 215 do Grupo Medicado e 192 do Grupo Controle. Houve 56 casos que foram excluídos do estudo por impossibilidade de seguimento em função de mudanças de endereço e não conformidade com os critérios de inclusão (Figura 1). As características demográficas e sociais do grupo recrutado e do grupo que completou o período de observação foram similares. Os resultados descritos a seguir referem-se aos 407 casos que completaram o estudo.

Observou-se que $62 \%$ tinham entre 20 e 29 anos de idade, sendo a média de idade de 26,9 anos. A maioria dos casos (57\%) não tinha filhos. A distribuição percentual dos casos por idade, situação conjugal e número de filhos está detalhada na Tabela 1 . Em relação à cor/raça, 55\% eram pardas, $34 \%$ brancas, $9 \%$ negras e $2 \%$ asiáticas. Um pouco mais de três quartos das participantes concluíram o ensino médio ou tinham escolaridade maior. Das mulheres com história de gravidez anterior ao estudo, 52\% relataram pelo menos um episódio de abortamento.

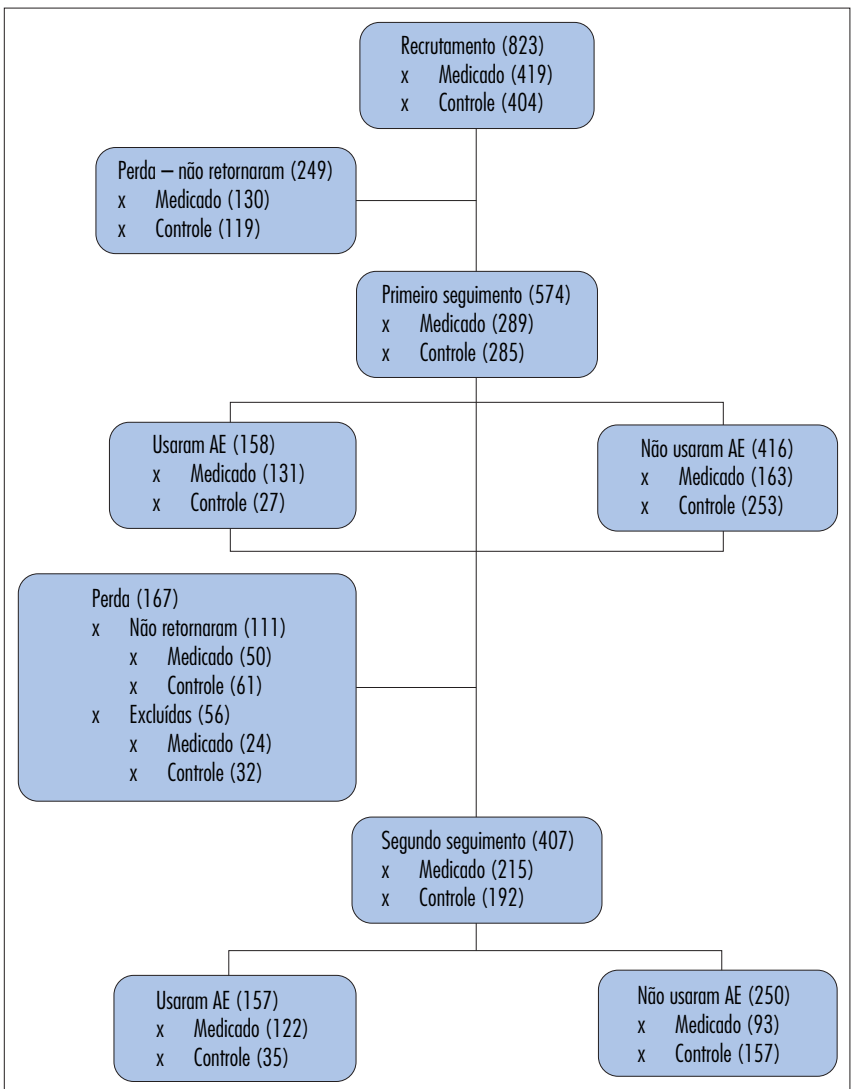

Figura 1 - Número de casos recrutados e que completaram as visitas de seguimento, por grupo de estudo. $A E=$ anticoncepção de emergência.

Tabela 1 - Distribuição percentual das mulheres que completaram 0 estudo por idade, situação conjugal e número de filhos vivos

\begin{tabular}{lc}
\hline Características & Porcentagem \\
\hline Idade & \\
$<20$ anos & 9,5 \\
$20-29$ & 62,0 \\
$30-34$ & 13,5 \\
35 e mais & 15,0 \\
Siłuaç̃o conjugal & \\
$\quad$ Solteira & 56,8 \\
Casada & 29,5 \\
Unicão consensual & 12,5 \\
Separada/divorciada & 1,2 \\
Número de fillhos vivos & \\
$\quad$ Não fem fillhos & 56,6 \\
1 fillho & 27,7 \\
2 fillhos & 12,3 \\
3 fillhos e mais & 3,4 \\
Número médio de fillhos vivos & 1,4 \\
Total & 100,0 \\
Número & 407 \\
\hline
\end{tabular}


Tabela 2 - Distribuição percentual das mulheres, segundo uso e frequiência de uso da AE, por grupo de estudo

\begin{tabular}{lccc}
\hline & \multicolumn{3}{c}{ Grupos de estudo } \\
\cline { 2 - 4 } & Medicado & Controle & Total \\
\hline Uso da AE* & & \\
Usou & 56,7 & 18,2 & 38,6 \\
Não usou & 43,3 & 81,8 & 61,4 \\
$\quad$ Tołal & 100,0 & 100,0 & 100,0 \\
$\quad$ Número & 215 & 192 & 407 \\
Freqüência de uso** & & & \\
$\quad 1$ vez & 33,3 & 54,8 & 37,6 \\
2 vezes & 26,2 & 29,0 & 26,8 \\
3 vezes & 22,2 & 9,7 & 19,7 \\
$\quad 4$ vezes ou mais & 18,3 & 6,5 & 15,9 \\
Tołal & 100,0 & 100,0 & 100,0 \\
Número & 122 & 35 & 157 \\
\hline
\end{tabular}

${ }^{*} p<0,000 ;{ }^{* *} p<0,05$.

Tabela 3 - Distribuição percentual das mulheres que usaram AE, segundo o uso de métodos anticoncepcionais no recrutamento (antes) e no final do estudo, por grupo

\begin{tabular}{|c|c|c|c|c|c|c|}
\hline \multirow{3}{*}{$\begin{array}{l}\text { Uso de métodos } \\
\text { anticoncepcionais }\end{array}$} & \multicolumn{6}{|c|}{ Grupo de estudo } \\
\hline & \multicolumn{2}{|c|}{ Medicado } & \multicolumn{2}{|c|}{ Controle } & \multicolumn{2}{|c|}{ Total } \\
\hline & Antes & Final & Antes & Final & Antes & Final \\
\hline \% não usando método & 12,3 & $3,2^{*}$ & 5,7 & $9,7^{\star \star}$ & 10,8 & 4,5 \\
\hline$\%$ usando algum método & 87,7 & 96,8 & 94,3 & $90,3^{* \star *}$ & 89,2 & 95,5 \\
\hline Injetável & 7,4 & 9,5 & 2,9 & 0,0 & 6,4 & 7,6 \\
\hline Pílula & 22,1 & 24,6 & 28,6 & 22,6 & 22,5 & 24,2 \\
\hline Preservativo masculino & 56,6 & 59,5 & 62,8 & 67,7 & 58,0 & 61,2 \\
\hline Coito interrompido & 1,6 & 3,2 & 0,0 & 0,0 & 1,3 & 2,5 \\
\hline Total & 100,0 & 100,0 & 100,0 & 100,0 & 100,0 & 100,0 \\
\hline Número & 122 & 122 & 35 & 35 & 57 & 157 \\
\hline
\end{tabular}

${ }^{*} \mathrm{p}=0,001 ;{ }^{* *} \mathrm{p}=0,32 ;{ }^{* * *} \mathrm{p}=0,32$.

A maior parte dos casos (61\%) não fez uso da AE. A AE foi utilizada por $39 \%$ das mulheres em algum momento durante o estudo, sendo 57\% do Grupo Medicado e 18\% do Grupo Controle (Tabela 2). Em 98\% das vezes, utilizouse o produto específico contendo levornogestrel.

Constatou-se que, no Grupo Medicado, houve uma maior frequiência de uso da AE em comparação ao Grupo Controle. A proporção de mulheres que utilizaram três vezes e quatro ou mais vezes foi superior no Grupo Medicado (22 e $18 \%$, respectivamente) do que no Grupo Controle (10 e $7 \%$, respectivamente). A análise da fonte de obtenção do método demonstra que a $\mathrm{AE}$ utilizada foi obtida em $83 \%$ dos casos nas clínicas Bemfam e em $16 \%$ nas farmácias. A grande maioria das mulheres do Grupo Medicado (96\%) relatou as clínicas Bemfam como fonte de obtenção do método. Entre as participantes do Grupo Controle que fizeram uso da $\mathrm{AE}$, quase dois terços $(65 \%)$ compraram o método na farmácia, apesar de terem sido informadas que poderiam recorrer às clínicas. As principais razões apontadas para o uso da AE durante o estudo foram: relação sexual sem proteção anticoncepcional $(48 \%)$ e falha no uso de outro método (46\%). Houve uma diferença significativa $(\mathrm{p}<0,05)$ no uso da AE pelas mulheres com história de abortamento (61\%), em comparação com mulheres sem história de abortamento (39\%).

Entre as mulheres que utilizaram a $\mathrm{AE}$ durante o estudo, $73 \%$ usaram o método até um dia após a relação sexual na qual perceberam risco de engravidar, $17 \%$ até dois dias, $8 \%$ até três dias e $2 \%$ quatro ou mais dias após. No Grupo Medicado, o uso foi feito, em $42 \%$ dos casos, até 12 horas após a relação sexual desprotegida, enquanto este percentual, no Grupo Controle, foi de $28 \%$.

Foram relatados efeitos colaterais por $21 \%$ das mulheres que usaram AE, sendo mais comuns náusea (64\%), cefaléia $(33 \%)$ e sensibilidade mamária (12\%). Não foi registrado nenhum caso de gravidez durante o estudo.

O comportamento contraceptivo da população investigada foi feito por meio da comparação da prevalência de uso de métodos anticoncepcionais em dois momentos distintos: no recrutamento e na segunda visita de seguimento. Observou-se um aumento na prevalência do uso regular de métodos anticoncepcionais, que, em média, passou de 89 para $96 \%$ ( $\mathrm{p}=0,002$, no teste de McNemar). No Grupo Medicado, observou-se um aumento na prevalência de uso da anticoncepção de $88 \%$ no recrutamento para $97 \%$ no final do estudo ( $\mathrm{p}=0,001$, no teste de McNemar). $\mathrm{O}$ aumento da prevalência de uso da anticoncepção neste grupo foi resultado do aumento de uso de todos os métodos citados pelas mulheres (Tabela 3). O declínio verificado no Grupo Controle não teve significância estatística ( $\mathrm{p}=0,32$, no teste de McNemar).

\section{Discussão}

A maior parte dos casos que completaram o estudo eram mulheres jovens, solteiras e com nível médio de escolaridade. Um dado relevante é o expressivo percentual de mulheres com relato de pelo menos um abortamento antes de ingressar no estudo (52\%), indicando alta vulnerabilidade para gravidez não planejada.

Apesar de terem informação e acesso gratuito à $\mathrm{AE}$, a maioria dos casos (61\%) não a utilizou no período de oito meses de observação.

Entre as mulheres que usaram o método (39\%), o uso foi maior no Grupo Medicado do que no Grupo Controle, 57 e $18 \%$, respectivamente. O uso repetido foi também mais freqüente no Grupo Medicado. De especial relevância é a observação de que o uso do método foi mais precoce no Grupo Medicado: 42\% usaram AE até 12 horas após a relação sexual desprotegida, enquanto, no Grupo Controle, este percentual foi de $28 \%$. Desde que o método é tanto mais efetivo quanto mais precocemente depois da relação sexual desprotegida for usado, a entrega antecipada favoreceu o uso mais precoce do método. A maior parte dos 
casos do Grupo Controle que usou AE obteve o método em farmácias. A não disponibilidade do método em mãos no momento da relação sexual desprotegida pode ter influenciado no menor uso do Grupo Controle.

A preocupação de que o uso da AE levaria a um relaxamento do uso regular de anticoncepcionais não se confirmou. Pelo contrário, houve aumento significativo de uso da anticoncepção regular no Grupo Medicado, inclusive do uso de preservativos, e uma redução não significativa no conjunto de métodos do Grupo Controle. Ainda assim, o uso de preservativos no Grupo Controle passou de 63 para 68\%. Algumas hipóteses para explicar este aumento do uso da anticoncepção regular são o reforço da orientação anticoncepcional e para prevenção de DST/HIV para as participantes do estudo e uma maior percepção do risco para gravidez não planejada. Os resultados do estudo confirmam dados de estudos similares realizados em outros países ${ }^{11-14,16}$.

Em uma revisão completa dos ensaios clínicos publicados sobre entrega antecipada de AE e sua relação com as taxas de gravidez, DST e comportamento sexual e contraceptivo, foram selecionados oito estudos, representando 6.389 casos nos Estados Unidos, China e Índia ${ }^{17}$. A entrega antecipada não reduziu as taxas de gravidez não planejada, apesar da maior freqüência de uso e do uso mais precoce com a entrega antecipada. Não houve aumento de DST nem da ocorrência de relações sexuais desprotegidas. Não houve mudança no padrão de uso de métodos anticoncepcionais. A utilização de preservativos foi similar entre as mulheres que receberam e as que não receberam o método antecipadamente.

O presente estudo apresenta algumas limitações. Primeiramente, a população estudada refere-se a um segmento específico: mulheres atendidas em serviços de saúde reprodutiva da rede privada em localidades urbanas das regiões Nordeste e Sudeste do Brasil. Além disso, há um expressivo percentual de usuárias de preservativos masculinos em ambos os grupos, decorrente do amplo trabalho de prevenção às DST/HIV realizado nas clínicas Bemfam. Dessa forma, os resultados não podem ser generalizados para a população como um todo. Em segundo lugar, é importante mencionar que não está claro o viés que possa ter ocorrido nas perdas ocasionadas pelas visitas de seguimento $(50,5 \%$ de perda do recrutamento à segunda visita de seguimento). Entretanto, deve-se considerar que as taxas de perda foram próximas às de outros estudos e comparações das distribuições percentuais de diversas características da população estudada, segundo o Grupo Medicado e o Grupo Controle ou segundo a amostra inicial (constituída pelas mulheres recrutadas) comparada à amostra final (constituída por aquelas que finalizaram o estudo), revelaram-se muito similares. Outro aspecto a ser mencionado é que o estudo utiliza informação retrospectiva (características sociodemográficas, história reprodutiva, uso da anticoncepção etc.), o que, devido a sua natureza, poderia estar sujeita a erro de memória.

Apesar das diferenças culturais e na prestação de serviços, o padrão de uso da $\mathrm{AE}$ com o acesso facilitado tem se mostrado similar em diversos países. A preocupação dos profissionais quanto ao abandono da anticoncepção regular e do uso de preservativos não foi confirmada por nenhum dos ensaios clínicos realizados até o momento. A divulgação destas pesquisas é fundamental para a redução das barreiras médicas à prescrição de $\mathrm{AE}$.

As pesquisas mais recentes sobre o uso da AE têm identificado outras questões relevantes, relacionadas à não redução das taxas de gravidez não planejada, apesar do maior uso da AE. Uma questão recorrente tem sido a não utilização de $\mathrm{AE}$ em situações de risco ${ }^{17}$. Nelson ${ }^{10}$ analisou as barreiras ao uso de preservativos e AE. Das 95 mulheres que tiveram pelo menos uma relação sexual desprotegida nas duas semanas que antecederam a avaliação, $37(39 \%)$ relataram uso da AE pelo menos uma vez. Os motivos alegados para o não uso foram similares aos apontados para o não uso de preservativos: percepção de não estar em risco porque realizaram coito interrompido ou porque pensaram não estar no período fértil.

A maior percepção de risco pode explicar o fato de que, no nosso estudo, a utilização de $\mathrm{AE}$ tenha sido significativamente maior entre as mulheres com história de abortamento.

O conhecimento acumulado com os diversos estudos apresentados nos permite inferir que o acesso à informação e aos produtos para AE deve ser ampliado significativamente. Com uma orientação consistente, as mulheres podem fazer uso da $\mathrm{AE}$ nos casos indicados, sem deixar de lado o uso regular de anticoncepcionais e a proteção contra DST.

No entanto, há desafios a serem enfrentados. Por que, tendo acesso facilitado à informação e ao método, mulheres que têm relações sexuais desprotegidas não fazem uso da AE? Que fatores levam ao não uso nos diferentes grupos populacionais? Quais são as intervenções comportamentais mais efetivas para que as mulheres reconheçam a própria vulnerabilidade à gravidez não planejada? São necessários estudos que abordem estas questões e que contemplem distintos segmentos populacionais como, por exemplo, mulheres usuárias dos serviços da rede pública de saúde.

\section{Agradecimentos}

Agradecemos às Fundações Bergstrom e West Wind, pelo apoio financeiro, e à Ângela Heimburger e à Federação Internacional de Planejamento Familiar, pelo apoio técnico para a implementação do estudo. 


\section{Referências}

1. Costa NFP, Almeida M, organizadores. Normas técnicas em anticoncepção. 2a ed. Rio de Janeiro: Bemfam; 2007.

2. Ministério da Saúde. Secretaria de Atenção à Saúde. Departamento de Ações Programáticas Estratégicas. Área Técnica de Saúde da Mulher. Anticoncepção de emergência: perguntas e respostas para profissionais de saúde. Brasília: MS; 2005.

3. Heimburger A, Gras C, Guedes A. Expanding access to emergency contraception: the case of Brazil and Colombia. Reprod Health Matters. 2003; 11 (21):150-60.

4. Schiappacasse V, Diaz S. Access to emergency contraception. Int J Gynaecol Obstet. 2006;94(3):301-9.

5. Galvão L, Diaz J, Diaz M, Osis M, Clark S, Ellertson C. Anticoncepção de emergência: conhecimento, atitudes e práticas entre ginecologistasobstetras no Brasil. Perspect Int Plan Fam. 2001 ; (N Esp.):20-3, 45.

6. Hardy EE, Duarte GA, Osis MD, Arce XE, Possan M. Anticoncepção de emergência no Brasil: facilitadores e barreiras. Cad Saúde Pública. 2001;17(4):1031-5.

7. Diaz S, Hardy E, Alvarado G, Ezcurra E. Aceitabilidade da anticoncepção de emergência no Brasil, Chile e México: 1 . Percepções sobre as pílulas de anticoncepção de emergência. Cad Saúde Pública. 2003;19(5):1507-17.

8. Diaz S, Hardy E, Alvarado G, Ezcurra E. Acceptability of emergency contraception in Brazil, Chile, and Mexico. 2 - Facilitating factors versus obstacles. Cad Saúde Pública. 2003;19(6):1729-37.

9. Figueiredo R, Andalaft Neto J. Uso de contracepção de emergência e camisinha entre adolescentes e jovens. Rev SOGIA-BR. 2005:6(2):1-11.
10. Nelson AL. Recent use of condoms and emergency contraception by women who selected condoms as their contraceptive method. Am J Obstet Gynecol. 2006;194(6):1710-5.

11. Ellertson C, Ambardekar S, Hedley A, Coyaji K, Trussell J, Blanchard K. Emergency contraception: randomized comparison of advance provision and information only. Obstet Gynecol. $2001 ; 98(4): 570-5$

12. Jackson RA, Schwarz EB, Freedman L, Darney P. Advance supply of emergency contraception: effect on use and usual contraception -a randomized trial. Obstet Gynecol. 2003;102(1):8-16.

13. Harper CC, Cheong M, Rocca CH, Darney PD, Raine TR. The effect of incresed access to emergency contraception among young adolescents. Obstet Gynecol. 2005;106(3):483-91

14. Marston C, Meltzer H, Majeed A. Impact on contraceptive practice of making emergency hormonal contraception available over the counter in Great Britain: repeated cross sectional surveys. BM. 2005;331(7511):271.

15. Siegel S. Estatística não-paramétrica: para as ciências do comportamento. São Paulo: McGraw-Hill do Brasil; 1975.

16. Rocca CH, Schwarz EB, Stewart FH, Darney PD, Raine TR, Harper CC. Beyond access: acceptability, use and nonuse of emergency contraception among young women. Am J Obstet Gynecol. 2007; 196(1):29.e 1-6.

17. Polis CB, Schaffer K, Blanchard K, Glasier A, Harper CC, Grimes DA. Advance provision of emergency contraception for pregnancy prevention (full review). Cochrane Database Syst Rev. 2007;(2):CD005497. 